\title{
Assessment of physical function using the developed device - Evaluations of the body function of the first evaluation device and prevention of bedsore using the cushion developed
}

\author{
Kazuhiko Yamashita ${ }^{1}$, Shigeo Takizawa ${ }^{2}$ \\ ${ }^{1}$ Ryotokuji University, ${ }^{2}$ Biophilia Institute
}

\section{Introduction}

The population rate of the elderly is steadily increasing in Japan, where $28.7 \%$ of the population represents a super-aging society. The population of some regions will decrease by $40 \%$ within the next 20 years, and the youth population will also decrease. Because the population composition is expected to remain the same for some time, there is an urgent need to develop and apply advanced technology in taking care of the elderly. Senior citizens tend to become bedridden, whether at home or in facilities and hence, are more prone to bedsores. Bedsores are caused by increased forces applied

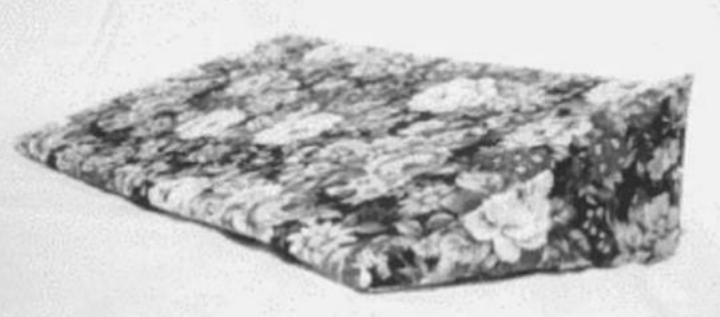

Fig.1 Appearance of five-side cushion to the sacrum and other protruding bones, and they can be mitigated by dispersing the pressure applied to these areas. The aim of this study is to develop a pressure-dispersing cushion and verify the effects of the pressure-dispersing cushion on the reduction of bedsores.

\section{Development of cushion for bedsore prevention}

Figure 1 shows the developed cushion. This cushion has five sides and is created by flattening the vertices of a triangle. The cushion is made of urethane. Its outer dimensions are as follows: $420 \mathrm{~mm}$ width, 100 and 10

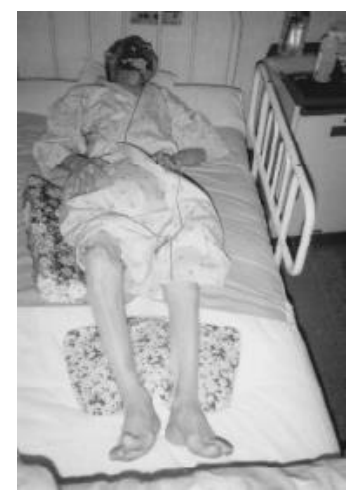

Fig. 2 fFive ${ }^{-}$-side cushioning to prevent heel sores$\mathrm{mm}$ height, $225 \mathrm{~mm}$ bottom depth, and the sharp part is positioned at $22.7^{\circ}$.

A patient was paralyzed owing to cerebrovascular disease. This cushion was used to maintain the posture while the patient was seated to decrease the pressure on the parts where bedsores may form.

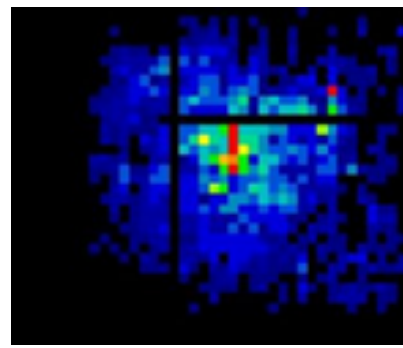

a. eCushion is not used $\bar{F}$ ig. 3 Results of pressure measurement 


\section{Methods}

A pressure distribution measurement system was used to measure the body forces applied to the cushion by the user, with the focus on the peak and average values near the sacrum and ischium (Figure 2). The resolution of the sensor was $1 \mathrm{~cm}$.

\section{Results}

The results of the experiment are shown in Figure 3. Parts a and b show the results when the cushion is and is not in use, respectively. The cushion reduced the peak pressure.

The local peak pressure must be below $200 \mathrm{mmHg}$ and requires a shift in position within $2 \mathrm{~h}$ to prevent bedsores. The developed cushion facilitates changing the position while decreasing the peak pressure, and thus, is effective for preventing bedsores.

\section{Analysis of lower limb Exercise by the Motivative Exercise}

\section{Introduction}

With a rapidly ageing society, the number of elderly people requiring nursing care is increasing. In particular, elderly patients with fallrelated fractures or cerebrovascular diseases, among others, require nursing care, and rehabilitation is considered vital in such cases. Currently, in the rehabilitation field, a small number of physical therapists are dealing with a large number of patients, making it difficult to secure a sufficient rehabilitation time for each patient. It is important for the patients to not only move passively during their paid treatment sessions with the physical therapist, but also exercise after rehabilitation, in their own rooms, to increase the effectiveness of rehabilitation. Additionally, if they are

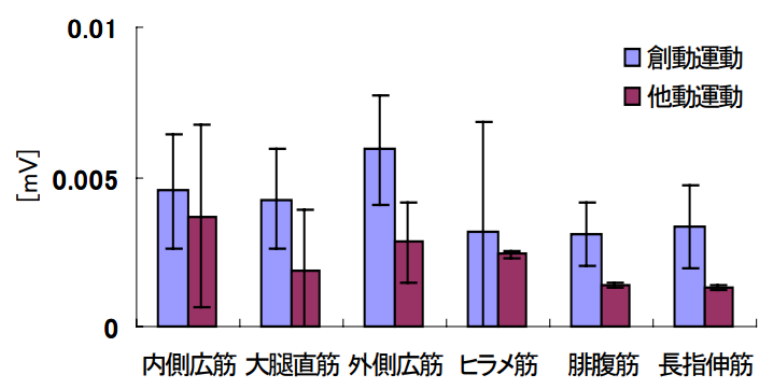

Fig. 1. Extension of the knee joint on the affected side unable to move after being discharged, the rehabilitation effects are reduced. Thus, there is a need for simple and effective rehabilitation tools that enable patients to continually be active at home.

Motivative exercise was developed for this purpose, and it has been reported that it is highly effective in recapturing the function of walking. However, muscular activity while moving 
during motivative exercise has not been sufficiently researched. This study aims to investigate muscular activity as a result of motivative exercise and estimate its rehabilitation benefits.

\section{Methods}

The subjects were two elderly people with paralysis in one leg caused by cerebrovascular disease. The muscles focused on in this study were those involved in the extension and bending of the knee joints and plantar flexion of the ankle joints. The soleus, gastrocnemius, extensor digitorum longus, rectus femoris muscle, vastus lateralis, and vastus medialis were measured on the healthy side and injured side of the subjects.

\section{Results}

The electromyography (EMG) results of the muscular activity of one of the test subjects are depicted in Figures 1 and 2. Muscular exercise with knee joint extension and ankle joint bending motion was $45 \%$ higher on the injured side with motivative exercise than with passive movement. Similarly, the knee bending movement and ankle movement dorsal motion muscular activity was 35\% higher in the vastus lateralis and $162 \%$ higher in extensor digitorum longus.

The motivative movement range was $11.4 \mathrm{~cm}$ in the knee extension direction and $27 \mathrm{~cm}$ in the bending direction. The results of the EMG measurement and movement range indicated that there was sufficient knee and ankle joint movement in motivative exercise. The above results suggest that exercise that moves the leg section of the injured side using that of the healthy side activates the muscular activity of the injured side and is effective in improving the muscles required for walking. 